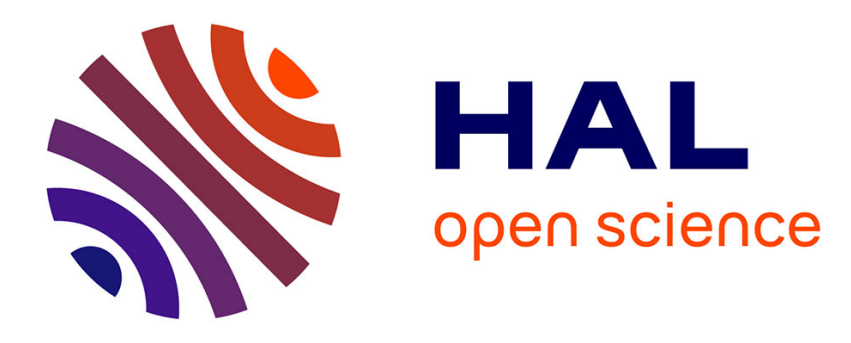

\title{
UAV-UGV Cooperation For Objects Transportation In An Industrial Area
}

El Houssein Chouaib Harik, François Guérin, Frédéric Guinand, Jean-François Brethé, Hervé Pelvillain

\section{- To cite this version:}

El Houssein Chouaib Harik, François Guérin, Frédéric Guinand, Jean-François Brethé, Hervé Pelvillain. UAV-UGV Cooperation For Objects Transportation In An Industrial Area. ICIT 2015. 2015 IEEE International Conference on Industrial Technology, Mar 2015, Séville, Spain. pp.547-552, 10.1109/ICIT.2015.7125156 . hal-01141993

\section{HAL Id: hal-01141993 https://hal.science/hal-01141993}

Submitted on 14 Apr 2015

HAL is a multi-disciplinary open access archive for the deposit and dissemination of scientific research documents, whether they are published or not. The documents may come from teaching and research institutions in France or abroad, or from public or private research centers.
L'archive ouverte pluridisciplinaire HAL, est destinée au dépôt et à la diffusion de documents scientifiques de niveau recherche, publiés ou non, émanant des établissements d'enseignement et de recherche français ou étrangers, des laboratoires publics ou privés. 


\title{
UAV-UGV Cooperation For Objects Transportation In An Industrial Area
}

\author{
El Houssein Chouaib Harik ${ }^{1}$, Student Member, IEEE, François Guérin ${ }^{2}$, Frédéric Guinand ${ }^{1}$, \\ Jean-François Brethé ${ }^{2}$, Member, IEEE, Hervé Pelvillain ${ }^{3}$
}

\begin{abstract}
We present in this paper a decentralized multirobot (aerial and ground) cooperation scheme for objects transportation. A team of ground mobile robots guided by a drone and a human operator moves in a coordinated way keeping a predefined formation in order to carry objects (tools, gas masks,...) in unsafe industrial areas. One ground mobile robot (leader) navigates among obstacles thanks to the waypoints provided by the drone and the human operator. The other ground mobile robots (followers) use a predictive vision based target tracking controller to keep a certain distance and bearing to the leader.
\end{abstract}

\section{INTRODUCTION}

\section{A. Motivation and related works}

Multi-robot cooperation attracts increasingly the attention of researchers, essentially for the numerous advantages that brings the deployment of a multi-robot system to perform a specific task. It brings the problems to a simpler level when the task complexity is too high to be performed by a single-robot to accomplish [1]. Nevertheless, multi-robot cooperation raises different challenges, in particular, the resolution of problems related to perception, decision and action [2]. We can find in the literature a huge amount of works related to multi-robot systems, surveys can be found in [3], [4], and [5].

A distinguished type of multi-robot cooperation is AirGround-Cooperation (AGC), where Unmanned Aerial Vehicles (UAVs) and Unmanned Ground Vehicles (UGVs) work in a cooperative way in order to perform a given task. The main advantage that can be drawn from AGC is the complementary skills provided by each type to overcome the specific limitations of the others (payload, perception, velocities, computational abilities, etc...), endowing a complete system to achieve a specific mission with higher efficiency and better delays. Moreover, deploying UAVs and UGVs in a single mission allows to envisage a wide range of applications.

In [6], an earthquake-damaged building scenario is used as an experimental field for providing a 3D map of the top three floors using a team of UAVs and UGVs. The main purpose of this work is to provide an insight of the degree of damage inside that building, and the team of robots (one UGV equipped with a laser rangefinder, one UAV also equipped with a laser rangefinder and a RGBD sensor) was

\footnotetext{
${ }^{1}$ LITIS, University of Le Havre. 25 Rue Philippe Lebon, 76600 Le Havre Cedex (France) - (el-houssein-chouaib.harik,frederic.guinand)@univlehavre.fr

${ }^{2}$ GREAH, University of Le Havre. 75, Rue Bellot, 76058 Le Havre Cedex (France) - (francois.guerin,jean-francois.brethe)@univ-lehavre.fr

${ }^{3}$ IUT GEII, University of Le Havre. Rue Boris Vian, 76610 Le Havre Cedex (France) - herve.pelvillain@univ-lehavre.fr
}

able to fulfill the task (to map the three floors) in a relatively short period of time (one afternoon). Similar works using AGC for mapping missions can be found in [7], [8], [9], [10]. Another application of AGC is cooperative navigation, where usually the UAV acts like a remote sensor or a flying eye [11]. In this work the authors use a UAV that flies ahead the UGV to provide geo-referenced 3D geometry in order to navigate safely avoiding obstacles (holes and ditches). The experimental results were threefold: the first experimentation consists in using only onboard sensors of the UGV to navigate in the area. In the second experimentation the UGV uses a traversability map that was already captured by the UAV and its onboard sensors. The third experimentation is similar to the second one with difference of adding new obstacle to the environment that has not been mapped before. The main scenario where the UAV flies ahead the UGV was validated through simulations, but some experimentations were carried out with the UAV manually piloted. A follow-up of the previous work can be found in [12]. The authors improved mainly their perception system, the UGV is still provided with prior maps taken from the UAV. Another approach can be found in [13] to overcome the UGV sensors limitations to detect negative obstacles (holes, cliffs, etc...). An aerial unmanned helicopter is used to gather data of an area, each point of data has three components: global spacial position, color measurements (RGB), and laser reflectance power measurement. In order to generate the obstacles cost map, a neural network classifier is used to classify the covered area into four classes (road, grass, tree, and building). The processed data is used as an input for a UGV planner to navigate in the covered area. Other related work using AGC for cooperative navigation and state estimation can be found in [14], [15], [16], [17], [18], [11],[19], [20], [21].

Extending these ideas, our team proposes a new scenario. We use a UAV to provide a global coverage for a team of UGVs during objects transportation. A human operator is in charge of supervising the team and providing waypoints to navigate safely in the area. For the team of UGVs we use a leader-follower approach. One UGV is designated at all times as a leader, while the other UGVs designated as the followers navigate in the area keeping a predefined distance and bearing to the leader using their embedded vision system. Visualbased formation has already largely been studied ([22], [23], [24]). The images acquired from an omnidirectional camera mounted on the leader allow to estimate the follower's pose. Thus the leader sends relative angular and linear velocities to the followers in order to maintain the desired formation. An 
omnidirectional camera offers a wide view, but it also needs important computational resources and complex algorithms (pose estimation). In our work, a pan-tilt colored camera is embedded on each follower, and a vision based tracking controller has been designed [25] to extract the distance and the bearing angle to a colored marker mounted on the leader.

\section{B. Contribution and organization}

Our contribution is twofold: in the previous related works, a first flight of the drone over the inspected area is always performed before the beginning of the mission. A traversability map is then processed to provide a trajectory to the UGV.

The first contribution of our work consists in providing a real-time navigation scheme. The UAV flies over the area to provide a global coverage, and to assist a UGV in real-time to navigate safely avoiding obstacles. To provide the real-time navigation scheme without the need to have a per-processed map, a human operator is included to select waypoints by monitoring the global and local coverages, making the architecture interactive, and applicable to real scenarios. For numerous reasons, monitoring, surveillance and inspection of industrial sites belonging to Seveso category (highly critical sites) cannot be performed without a constant and careful attention of human experts.

The second contribution concerns the visual-based formation. In the previous works, the leader is always responsible for observing the followers state. It sends the necessary linear and angular velocities of each follower to maintain a predefined configuration. We use in our work a leaderfollower approach, with the difference that each follower is considered as an independent entity. It uses its embedded color vision system to estimate the leader's pose (distance and bearing of the colored marker mounted on the leader) to regulate the linear and angular velocities accordingly. Thus no communication between the team members is required, what makes our proposed architecture does not need significant computational and communication requirements.

This paper is organized as follows: we present in section II the problem statement, and discuss the envisaged scheme. Section III presents the architecture design. We give in section IV some simulation and experimental results, and we conclude in section $\mathrm{V}$ the present work and discuss future perspectives.

\section{Problem Statement}

A UAV (drone) is used to guide among obstacles (Figure 1) a team of UGVs (mobile robots) to perform objects transportation tasks (tools, gas masks,...) in unsafe industrial areas. The video flow is sent continuously to the ground station from the camera mounted on the UAV. It is used in real time to provide localization information to a UGV (that we will designate as a leader) to navigate in the industrial area through the on-the-go waypoints selected by a human operator.

The other UGVs are called followers and are used to carry heavy loads. As the leader navigates to each next waypoint, these followers take the leader as a target and follow it keeping a certain separation distance and bearing in order to form a specific configuration that can be defined prior, or during the mission. In our study, we used two followers, but this number can be extended depending on the mission goals.

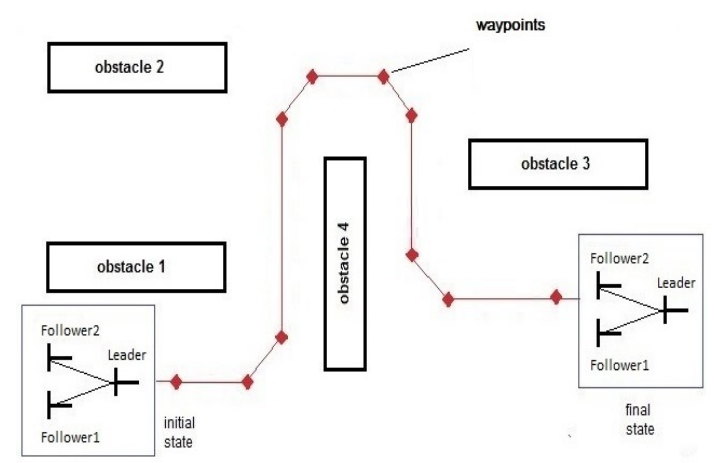

Fig. 1. Problem statement

\section{ARChitecture DESIGN}

\section{A. Hardware configuration}

We will consider in this work three similar non-holonomic, unicycle-like wheeled mobile robot (UGV) and a quadcopter drone (UAV) equipped with a color camera. The video flow is sent continuously to the ground station where it will be processed to extract the position and the orientation of the leader relative to the next waypoint. These information are then sent to the leader to correct its trajectory in real time using a non-linear kinematic controller.

\section{B. Overall architecture}

We can divide our architecture (Figure 2) in two layers: Drone-Leader layer, and Leader-Followers layer. We will explain in the next section the functionality as well as the controllers design of each layer.

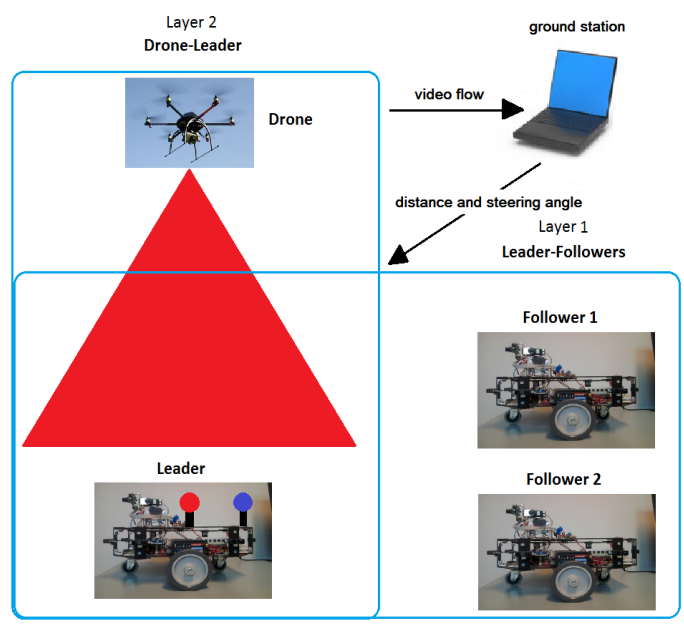

Fig. 2. Overall architecture 


\section{First layer: Leader-Followers}

In the first layer (leader-followers), the followers use their embedded vision system to track the leader keeping a predefined configuration (Figure 3 ). The embedded vision system is assumed to be located at a distance $L$ from $Y_{F}$, the $y$-axis of the UGV coordinate frame (Figure 4). The vision system outputs are the distance (range) $z$ and the orientation (bearing) $\theta$ of a follower relative to the moving target (leader).

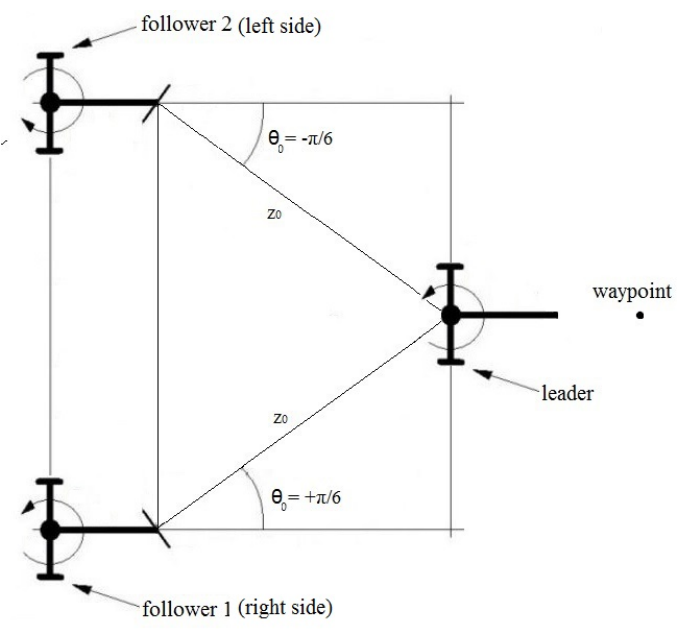

Fig. 3. Leader-follower configuration

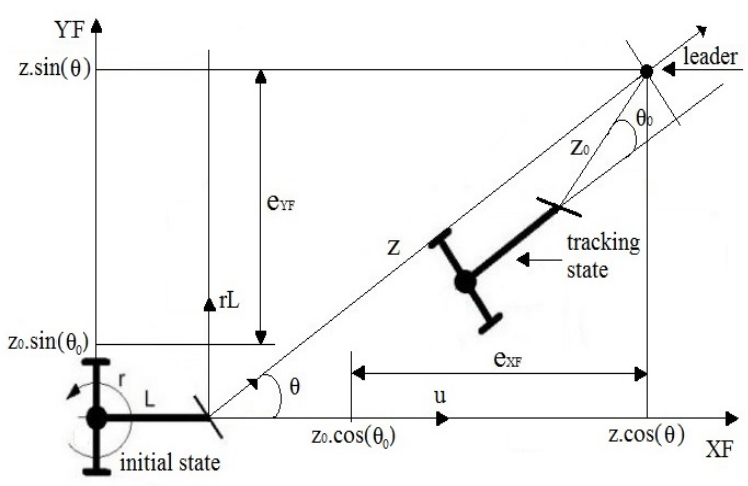

Fig. 4. Design of the follower controller

The control objective (Figure 4) consists in regulating the distance $z$ and the bearing angle $\theta$ as follows:

$$
z_{0}<\lim _{t \rightarrow \infty} z(t)<z_{1} \quad \lim _{t \rightarrow \infty} \theta(t)=\theta_{0}
$$

Where $z_{0}$ is the predefined separation distance, and $\theta_{0}$ is the predefined bearing angle. $\theta_{0}$ is $+\frac{\pi}{6}$ for the first follower (right), and $-\frac{\pi}{6}$ for the second follower (left) (Figure 3). As the leader moves at unknown velocities, and because of the non-holonomic constraints of the UGVs, the distance $z$ cannot be regulated to $z_{0}$ permanently, it has to be bounded with an upper allowable distance $z_{1}$.
1) Design of the non-linear kinematic controller: To fulfill the control objective (1), we consider the following errors $e_{X F}$ and $e_{Y F}$ corresponding to the differences between the initial and the tracking state:

$$
\begin{aligned}
& e_{X F}=z \cdot \cos (\theta)-z_{0} \cdot \cos \left(\theta_{0}\right) \\
& e_{Y F}=z \cdot \sin (\theta)-z_{0} \cdot \sin \left(\theta_{0}\right)
\end{aligned}
$$

The time derivatives of $e_{X F}$ and $e_{Y F}$ are given by:

$$
\begin{aligned}
& \dot{e}_{X F}=\dot{z} \cdot \cos (\theta)-z \cdot \dot{\theta} \cdot \sin (\theta) \\
& \dot{e}_{Y F}=\dot{z} \cdot \sin (\theta)+z \cdot \dot{\theta} \cdot \cos (\theta)
\end{aligned}
$$

$\dot{e}_{X F}$ and $\dot{e}_{Y F}$ depends on the UGV's velocities $(u, r)$ and the target's velocities along the $X_{F}$ and $Y_{F}$ axes, denoted as $T V_{X F}, T V_{Y F}$, thus (3) can be written as follows:

$$
\begin{array}{r}
\dot{e}_{X F}=T V_{X F}-u \\
\dot{e}_{Y F}=T V_{Y F}-r . L
\end{array}
$$

r. $L$ is the linear velocity of the point on the UGV at which the embedded vision system is located. The angle $\theta$ can vary between $-\pi / 2$ and $+\pi / 2$ due to mechanical restrictions. We propose the following stable error dynamic equations:

$$
\begin{aligned}
\dot{e}_{X F} & =-K . e_{X F} \\
\dot{e}_{Y F} & =-K . e_{Y F}
\end{aligned}
$$

Where $(K>0)$ is a proportional gain, substituting (2) and (3), this leads to:

$$
\begin{aligned}
T V_{X F}-u & =-K \cdot\left(z \cdot \cos (\theta)-z_{0} \cdot \cos \left(\theta_{0}\right)\right) \\
T V_{Y F}-r . L & =-K .\left(z \cdot \sin (\theta)-z_{0} \cdot \sin \left(\theta_{0}\right)\right)
\end{aligned}
$$

Since the target's velocities $\left(T V_{X F}, T V_{Y F}\right)$ are unknown, we propose the following non-linear kinematic controller:

$$
\begin{array}{r}
u=K \cdot\left(z \cdot \cos (\theta)-z_{0} \cdot \cos \left(\theta_{0}\right)\right) \\
r=K \cdot\left(z \cdot \sin (\theta)-z_{0} \cdot \sin \left(\theta_{0}\right)\right) / L
\end{array}
$$

2) Stability analysis: We consider the following Lyapunov candidate function:

$$
V=\frac{e_{X F^{2}}+e_{Y F^{2}}}{2} \quad(V>0)
$$

Its time derivative can be written as:

$$
\begin{gathered}
\dot{V}=e_{X F} \cdot \dot{e}_{X F}+e_{Y F} \cdot \dot{e}_{Y F} \\
\dot{V}=e_{X F} \cdot\left(T V_{X F}-K \cdot e_{X F}\right)+e_{Y F} \cdot\left(T V_{Y F}-K \cdot e_{Y F}\right)
\end{gathered}
$$

Thus, $\dot{V}<0$ if $\left|e_{X F}\right| \geq\left|\frac{T V_{X F}}{K}\right|$ and $\left|e_{Y F}\right| \geq\left|\frac{T V_{Y F}}{K}\right|$ so that the size of the tracking errors $\left|e_{X F}\right|$ and $\left|e_{Y F}\right|$ are uniformly ultimately bounded by $\left(\frac{\overline{T V} V_{X F}}{K}\right)$ and $\left(\frac{\overline{T V} V_{Y F}}{K}\right)$ respectively, where $\left(\overline{T V}_{X F}\right)$ and $\left(\overline{T V}_{Y F}\right)$ represent bounds on the target's velocity components satisfying $\left|T V_{X F}\right|<$ $\overline{T V}_{X F}$ and $\left|T V_{Y F}\right|<\overline{T V}_{Y F}$. These tracking errors can never become zero because the target velocities are unknown to the controller. However, large gain $K$ leads to small error bounds. That is why we are going to propose in the next section an automatic tuning of the proportional gain $K$ resting on the use of a predictive double exponential smoothing algorithm. 
3) DES algorithm: The Double Exponential Smoothing (DES) algorithm [26] has been implemented to smoothen the uncertain measurement given by the vision system. The DES algorithm runs approximately 135 times faster with equivalent prediction performances and simpler implementations compared to a Kalman filter.

The DES algorithm at time instant $n . T_{e}$, where $T_{e}$ is the sampling period and $n$ is the discrete-time index, implemented for both the distance $(z)$ and bearing angle $(\theta)$ measurements is given as follows:

$$
\begin{gathered}
S_{z_{n}}=\gamma_{z} \cdot z_{n}+\left(1-\gamma_{z}\right) \cdot\left(S_{z_{n-1}}+b_{z_{n-1}}\right) \\
b_{z_{n}}=\lambda_{z} \cdot\left(S_{z_{n}}-S_{z_{n-1}}\right)+\left(1-\lambda_{z}\right) \cdot b_{z_{n-1}} \\
S_{\theta_{n}}=\gamma_{\theta} \cdot \theta_{n}+\left(1-\gamma_{\theta}\right) \cdot\left(S_{\theta_{n-1}}+b_{\theta_{n-1}}\right) \\
b_{\theta_{n}}=\lambda_{\theta} \cdot\left(S_{\theta_{n}}-S_{\theta_{n-1}}\right)+\left(1-\lambda_{\theta}\right) \cdot b_{\theta_{n-1}}
\end{gathered}
$$

Where:

$z_{n}, \theta_{n}$ are the values of $z$ and $\theta$ at $n$th sample instant.

$S_{z_{n}}, S_{\theta_{n}}$ are the smoothed values of $z$ and $\theta$.

$b_{z_{n}}$ and $b_{\theta_{n}}$ are the trend values.

Equations (11) and (13) smooth the values of the sequence of measurements by taking into account the trend, whilst (12) and (14) smooth and update the trend. The $m(m>0)$ steps ahead forecast of the next position $(z)$ estimation is:

$$
F_{Z_{n+m}}=S_{Z_{n}}+m \cdot b_{Z_{n}}
$$

The initial values given to $S_{z_{n}}, S_{\theta_{n}}, b_{z_{n}}$ and $b_{\theta_{n}}$ are:

$$
S_{z_{1}}=z_{1}, S_{\theta_{1}}=\theta_{1}, b_{z_{1}}=z_{2}-z_{1}, b_{\theta_{1}}=\theta_{2}-\theta_{1}
$$

Usually, $\gamma(0 \leq \gamma \leq 1)$ is called the data smoothing factor and $\lambda(0 \leq \lambda \leq 1)$ is called the trend smoothing factor. A compromise has to be found for the values of $\gamma$ and $\lambda$. High values make the DES algorithm follow the trend more accurately whilst small values make it generate smoother results. By using the DES algorithm, the non-linear kinematic controller (7) becomes:

$$
\begin{array}{r}
u=K \cdot\left(S_{z_{n}} \cdot \cos \left(S_{\theta_{n}}\right)-z_{0} \cdot \cos \left(\theta_{0}\right)\right) \\
r=K \cdot\left(S_{z_{n}} \cdot \sin \left(S_{\theta_{n}}\right)-z_{0} \cdot \sin \left(\theta_{0}\right)\right) / L
\end{array}
$$

In (17), the smoothed values are used instead of the direct noisy measurements of $z$ and $\theta . F_{Z_{n+m}}$ the prediction of $z$ in (15) can now be used for the auto-tuning of the proportional gain $(K)$ in the non-linear kinematic controller as follows:

$$
\frac{d K}{d t}=\beta \cdot \operatorname{sign}\left(F_{Z_{n+m}}-z_{0}^{*}\right) \quad \text { with } \beta>0
$$

In discrete time (sampling period $T_{e}$ ), (18) can be written as:

$$
K_{n}=K_{n-1}+T_{e} \cdot \beta \cdot \operatorname{sign}\left(F_{Z_{n}}-z_{0}^{*}\right)
$$

The proportional gain $K$ increases or decreases stepwise. This auto-tuning allows to satisfy the control objective (1), since the distance $z$ can never be regulated to $z_{0}$ permanently due to the random motion of the target, the non-holonomic restrictions and the dynamic properties of the UGV. The value of $z_{0}^{*}$ is chosen according to equation (1) to satisfy the control objective (namely the allowable upper bound specification): $z_{0}<z_{0}^{*}<z_{1}$.

\section{Second layer: Drone-Leader}

A UAV equipped with a colored camera is used to provide a global coverage of the industrial area. It assists the UGV (leader) in navigation among obstacles. The UAV flies at a constant altitude, keeping the UGV (leader) in the center of the image plan (visual servoing). Waypoints are assigned in the image plan by a human operator according to the free-space navigation (Figure 5). The UGV (leader) navigates through the selected waypoints, followed continuously by the UAV (this work is under submission).

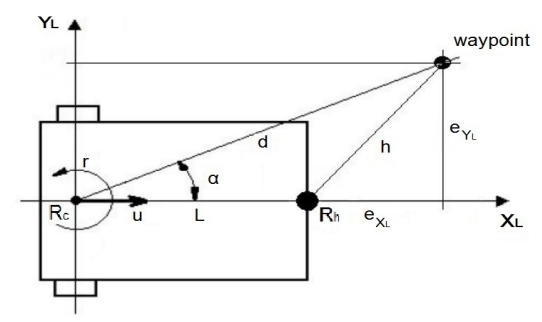

Fig. 5. Leader controller

Note that the classical pinhole model [27] is used to estimate the reference values of $d$ and $\alpha$ in the world frame. They were previously extracted in pixels (image processing). Odometry is used to estimate the UGV's current state to its next waypoint.

Let us consider (Figure 5) the following distance errors on $X_{L}$ and $Y_{L}$ axes respectively:

$$
\begin{array}{r}
e_{X_{L}}=d \cdot \cos (\alpha)-L \\
e_{Y_{L}}=d \cdot \sin (\alpha)
\end{array}
$$

The time derivative of the distance errors (20), which depends on both the leader's velocities $(u, r)$ and the target's velocities along the $X_{L}$ and $Y_{L}$ axes, denoted as $T V_{X_{L}}$ and $T V_{Y_{L}}$, are given by:

$$
\begin{aligned}
& \dot{e}_{X_{L}}=\dot{d} \cdot \cos (\alpha)-d \cdot \dot{\alpha} \cdot \sin (\alpha)=T V_{X_{L}}-u \\
& \dot{e}_{Y_{L}}=\dot{d} \cdot \sin (\alpha)+d \cdot \dot{\alpha} \cdot \cos (\alpha)=T V_{Y_{L}}-r L
\end{aligned}
$$

Where $r L$ corresponds to the linear velocity of $R_{h}$ (blue marker).

$T V_{X_{L}}$ and $T V_{Y_{L}}$ are null since the leader's target is a waypoint. We propose then the following stable error dynamic equations:

$$
\begin{gathered}
\dot{e}_{X_{L}}=-K \cdot e_{X_{L}}=-u \\
\dot{e}_{Y_{L}}=-K \cdot e_{Y_{L}}=-r . L
\end{gathered}
$$


Where $K>0$ is the proportional gain of the leader's controller. Note that for large values of the distance $d$, a saturation is implemented to consider the limitations of the electrical drives that control the WMR's wheels.

Starting from (22) we propose the following non-linear kinematic controller:

$$
\left\{\begin{array}{l}
u=K \cdot(d \cdot \cos (\alpha)-L) \\
r=K \cdot(d \cdot \sin (\alpha)) / L
\end{array}\right.
$$

Stability analysis can be studied starting from the same Lyapunov function (8), which shows that the stability of our proposed controller (23) is guaranteed.

\section{RESUlTS}

\section{A. Simulation results}

We will present in this section simulation results of the proposed architecture. We simulated a real-like scenario to navigate in the transportation area through the given waypoints. For each UGV (leader and followers), we have used the dynamic model described in [25] and [27]. Simulations have been carried out with Matlab-Simulink software (sampling period: $10 \mathrm{~ms}$ ). For the follower's non-linear kinematic controllers, we used the same parameters as in [25] except for the bearing angle $\left(\theta_{0}= \pm \pi / 6\right)$ and the distance $z_{0}=80 \mathrm{~cm}$. For the UGV's non-linear kinematic controller we used the following parameters: $L=18 \mathrm{~cm}, K=0.1$. The simulated scenario consists in giving waypoints to navigate in a specific area avoiding obstacles. Figure 6 shows the trajectories of each UGV.

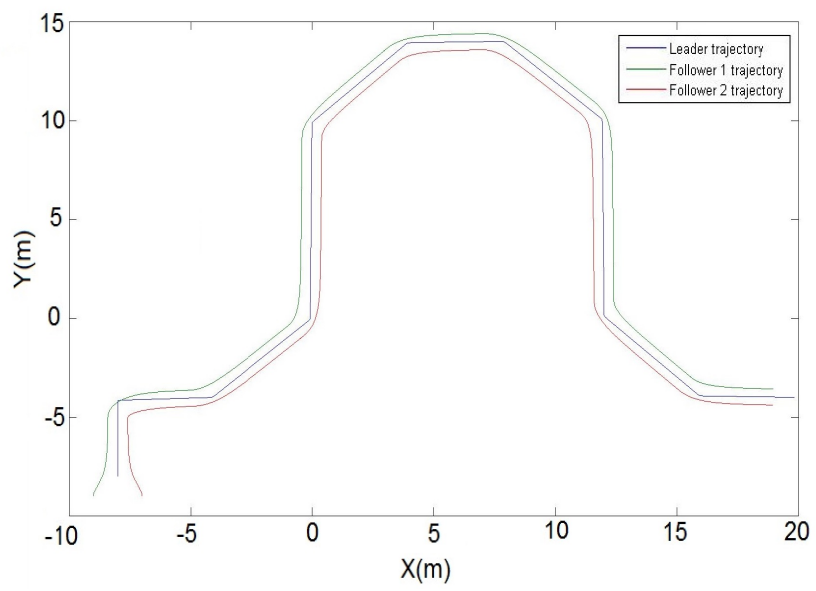

Fig. 6. Waypoints tracking

The simulation showed the efficiency of the developed architecture and the proposed controllers. The leader is able to follow the given waypoints and the followers are able to navigate using it as a target while keeping a predefined separation distance and bearing angle.

In figures 7,8,9, we notice that the mean distance between the UGVs is around $98 \mathrm{~cm}$. It corresponds to the addition of the given distance to be maintained between the UGVs $z_{0}=80 \mathrm{~cm}$ and $L=18 \mathrm{~cm}$, which confirms the efficiency of our developed controller (17). We can see (Figure 6) that the leader's trajectory is discontinuous. It stops at each waypoint to change its direction to the next one, which explains the peaks on figures $7,8,9$. They correspond to the moment when the leader changes its direction for a new waypoint, creating a sharp variation in the corresponding distance between him and the followers. Note that maintaining the distance between the three UGVs means the maintain of the desired angle $\theta_{0}$.

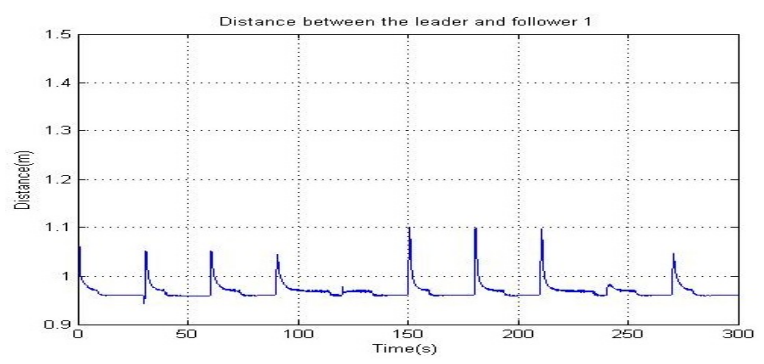

Fig. 7. Distance between the leader and the follower 1

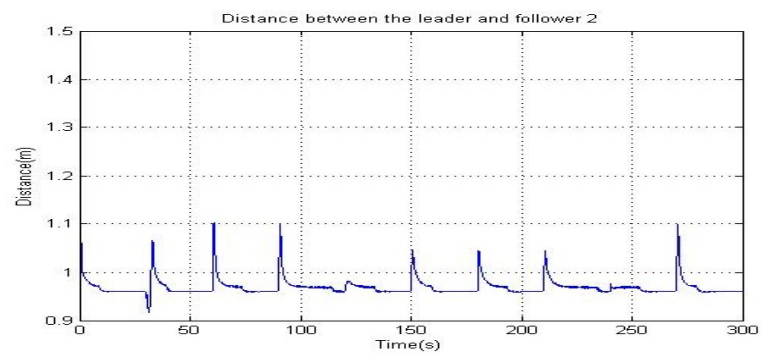

Fig. 8. Distance between the leader and the follower 2

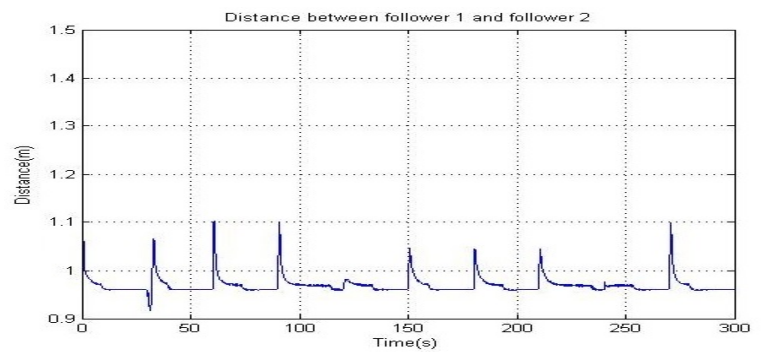

Fig. 9. Distance between the follower 1 and the follower 2

\section{B. Experimental results}

We created a user interface using the development environment Processing [28] running on the ground station, where a human operator can supervise in real time the UGVs through a video flow received continuously from the camera mounted beneath the UAV. The human operator can select waypoints in the image by a simple click on the desired position. The program extracts and send the distance $d$ and the orientation $\alpha$ to the leader via ZigBee module (IEEE 802.15.4) at a $50 \mathrm{~Hz}$ frequency (real time interrupt). Because we do not yet have access to position measurements in the inertial frame (using for example VICON MX system), the results concerning the leader-followers layer are limited to simulations trials only. 


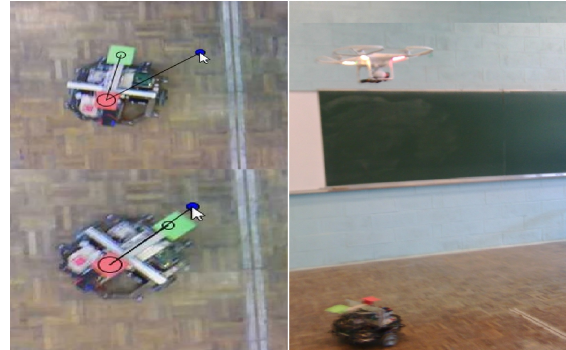

Fig. 10. Waypoint tracking experimentation

Figure 10 shows that the UGV, after clicking on the desired waypoint, used the bearing angle $\alpha$ and the distance $d$ received from the ground station, to navigate successfully to that point.

\section{CONCLUSION AND FUTURE WORK}

We introduced in this paper a control scheme for a multirobot system where a group of UGVs and a UAV work for a transportation task. One UGV is designated at all times to be the leader of a team of UGVs (followers). A UAV is used to provide a global coverage of the area allowing the leader to navigate safely in the transportation area through waypoints selected by a human operator. Each follower is considered as an independent entity. It navigates using a predictive vision based target tracking controller taking the leader as a target, what makes the communication and computational requirements kept at a lower level. The tracking algorithm can be improved by endowing the follower's controllers by the leader's velocity, which will limit the distance errors between the UGVs and thus will improve the proposed architecture for cooperatively transporting a single heavy load.

\section{REFERENCES}

[1] L. E. Parker, B. Siciliano, and O. Khatib. "Multiple Mobile Robot Systems". Springer Handbook of Robotics, 2008.

[2] Simon Lacroix and Guy Besnerais. "issues in cooperative air/ground robotic systems". In Makoto Kaneko and Yoshihiko Nakamura, editors, Robotics Research, volume 66 of Springer Tracts in Advanced Robotics, pages 421-432. Springer Berlin Heidelberg, 2011.

[3] Adrián Jiménez-González, Jose Ramiro Martinez-de Dios, and Anibal Ollero. Testbeds for ubiquitous robotics: A survey. Robotics and Autonomous Systems, 61(12):1487-1501, 2013.

[4] Zhi Yan, Nicolas Jouandeau, and Arab Ali Cherif. A survey and analysis of multi-robot coordination. International Journal of Advanced Robotic Systems, 10, 2013.

[5] Y Uny Cao, Alex S Fukunaga, and Andrew Kahng. Cooperative mobile robotics: Antecedents and directions. Autonomous robots, 4(1):7-27, 1997.

[6] Nathan Michael, Shaojie Shen, Kartik Mohta, Yash Mulgaonkar, Vijay Kumar, Keiji Nagatani, Yoshito Okada, Seiga Kiribayashi, Kazuki Otake, Kazuya Yoshida, et al. Collaborative mapping of an earthquakedamaged building via ground and aerial robots. Journal of Field Robotics, 29(5):832-841, 2012.

[7] Christian Forster, Matia Pizzoli, and Davide Scaramuzza. Air-ground localization and map augmentation using monocular dense reconstruction. In Intelligent Robots and Systems (IROS), 2013 IEEE/RSJ International Conference on, pages 3971-3978. IEEE, 2013.

[8] H Jin Kim, Rene Vidal, David H Shim, Omid Shakernia, and Shankar Sastry. A hierarchical approach to probabilistic pursuit-evasion games with unmanned ground and aerial vehicles. In Decision and Control, 2001. Proceedings of the 40th IEEE Conference on, volume 1, pages 634-639. IEEE, 2001
[9] René Vidal, Shahid Rashid, Cory Sharp, Omid Shakernia, Jin Kim, and Shankar Sastry. Pursuit-evasion games with unmanned ground and aerial vehicles. In Robotics and Automation, 2001. Proceedings 2001 ICRA. IEEE International Conference on, volume 3, pages 29482955. IEEE, 2001.

[10] M Ani Hsieh, Anthony Cowley, James F Keller, Luiz Chaimowicz, Ben Grocholsky, Vijay Kumar, Camillo J Taylor, Yoichiro Endo, Ronald C Arkin, Boyoon Jung, et al. Adaptive teams of autonomous aerial and ground robots for situational awareness. Journal of Field Robotics, 24(11-12):991-1014, 2007

[11] Anthony Stentz, Alonzo Kelly, Herman Herman, Peter Rander, Omead Amidi, and Robert Mandelbaum. Integrated air/ground vehicle system for semi-autonomous off-road navigation. Robotics Institute, page 18, 2002.

[12] Anthony Stentz, Alonzo Kelly, Peter Rander, Herman Herman, Omead Amidi, Robert Mandelbaum, Garbis Salgian, and Jorgen Pedersen. Real-time, multi-perspective perception for unmanned ground vehicles. AUVSI, 2003

[13] Boris Sofman, J Andrew Bagnell, Anthony Stentz, and Nicolas Vandapel. Terrain classification from aerial data to support ground vehicle navigation. Carnegie Mellon University. 2006.

[14] Matthias Faessler, Elias Mueggler, Karl Schwabe, and Davide Scaramuzza. A monocular pose estimation system based on infrared leds. International Conference on Robotics and Automation (ICRA), 2014.

[15] Donald K MacArthur and Carl D Crane. "Unmanned ground vehicle state estimation using an unmanned air vehicle". In Computational Intelligence in Robotics and Automation, 2007. CIRA 2007. International Symposium on, pages 473-478. IEEE, 2007.

[16] Rad Madhavan, Tsai Hong, and Elena Messina. Temporal range registration for unmanned ground and aerial vehicles. Journal of Intelligent and Robotic Systems, 44(1):47-69, 2005.

[17] Jae-Young Choi and Sung-Gaun Kim. Collaborative Tracking Control of UAV-UGV. World Academy of Science, Engineering and Technology, 71, 2012.

[18] Luiz Chaimowicz, Ben Grocholsky, James F Keller, Vijay Kumar, and Camillo $\mathrm{J}$ Taylor. "Experiments in multirobot air-ground coordination". In Robotics and Automation, 2004. Proceedings. ICRA'04. 2004 IEEE International Conference on, volume 4, pages 4053-4058. IEEE, 2004.

[19] Nicolas Vandapel, Raghavendra Rao Donamukkala, and Martial Hebert. Unmanned ground vehicle navigation using aerial ladar data. The International Journal of Robotics Research, 25(1):31-51, 2006.

[20] Nicolas Vandapel, Raghavendra R Donamukkala, and Martial Hebert. Quality assessment of traversability maps from aerial lidar data for an unmanned ground vehicle. In Intelligent Robots and Systems, 2003.(IROS 2003). Proceedings. 2003 IEEE/RSJ International Conference on, volume 1, pages 305-310. IEEE, 2003.

[21] Nicolas Vandapel, Raghavendra Donamukkala, and Martial Hebert. Experimental results in using aerial ladar data for mobile robot navigation. In Field and Service Robotics, pages 103-112. Springer, 2006.

[22] Christiano Couto Gava, Raquel Frizera Vassallo, Flavio Roberti, Ricardo Carelli, and Teodiano Freire Bastos-Filho. Nonlinear control techniques and omnidirectional vision for team formation on cooperative robotics. In Robotics and Automation, 2007 IEEE International Conference on, pages 2409-2414. IEEE, 2007.

[23] Gian Luca Mariottini, Fabio Morbidi, Domenico Prattichizzo, Nicholas Vander Valk, Nathan Michael, George Pappas, and Kostas Daniilidis. Vision-based localization for leader-follower formation control. Robotics, IEEE Transactions on, 25(6):1431-1438, 2009.

[24] F. Roberti, J. M. Toibero, R. Carelli, and R. F. Vassallo. Stable formation control for a team of wheeled mobile robots. Reunión de Trabajo en Procesamiento de la Información y Control, 2007.

[25] François Guerin, Simon G. Fabri, and Marvin K. Bugeja. "double exponential smoothing for predictive vision based target tracking of a wheeled mobile robot". In Decision and Control (CDC), 2013 IEEE 52nd Annual Conference on, pages 3535-3540, Florence, Italy, December 2013.

[26] Joseph J LaViola Jr. "an experiment comparing double exponential smoothing and kalman filter-based predictive tracking algorithms". In Virtual Reality, 2003. Proceedings. IEEE, pages 283-284. IEEE, 2003.

[27] François Guerin. "commande conjuguée d'un robot mobile, modélisation dynamique et vision artificielle", thèse de doctorat de l'université du havre - eue - isbn 978-613-1-54896-3. 2004.

[28] Processing. Processing 2, URL: http://www.processing.org/. 2014. 